\title{
Efficacy of A-type Botulinum Toxin on Children with Equinus Foot Classified by the Plantarflexor-knee Extension Couple Index: A Retrospective Study
}

\author{
Andrea Ancillao ${ }^{1,3 *}$, Manuela Galli ${ }^{1}$ and Claudia Condoluci ${ }^{2}$ \\ ${ }^{1}$ Department of Electronic, Information and Bioengineering, "Politecnico di Milano", Milano, Italy \\ 2IRCCS “San Raffaele Pisana”, San Raffaele SPA, Roma, Italy \\ ${ }^{3}$ Tyndall National Institute, "University College Cork", Cork, Ireland
}

\begin{abstract}
Background and Aim: Botulinum toxin is often adopted as a treatment for muscle spasticity and toe walking in children with several palsy. Efficacy and long-term effect of botulinum toxin are currently discussed in the literature as the effects may vary depending on the subject. The aim of this work was to study the efficacy of botulinum toxin treatment on toe-walkers with different walking patterns.

Methods: The analysis was conducted on children diagnosed with diplegia related to cerebral palsy. The walking patterns were classified according to the plantarflexor-knee extension couple index. Deviation in gait patterns and changes post-treatment were quantified by summary measures based on kinematics in order to evaluate the overall effect.

Outcomes: Three motor phenotypes were identified: (i) Bilateral true equinus, (ii) Bilateral jump gait, (iii) Asymmetric gait. Bilateral true equinus and bilateral jump gait subjects had a post-treatment improvement at the ankle joint but no improvement in the overall gait. The subjects with asymmetric gait did not benefit from the treatment.

Conclusions and Implications: This study showed that botulinum toxin had its main effect locally rather than on the overall gait. Both phenotypes of equinus foot (bilateral true equinus and bilateral jump gait) are good candidates for this treatment. The treatment of toe-walkers subjects with botulinum toxin is recommended only if a localized and temporary effect is desired.
\end{abstract}

Keywords: Botulinum toxin; Cerebral palsy; Equinus foot; Gait analysis; Rehabilitation; Toe walking

\section{Introduction}

Spastic Cerebral Palsy (CP) is a complex pathology of neurologic origin expressed by physical disabilities due to tone alterations of muscles. The equinus foot is a pathological condition that is commonly observed in children with CP. Such condition is characterized by a permanent plantar flexion that leads to toe walking and balancing issues [1]. The causes were identified in the status of permanent contraction or spasticity of the Triceps surae muscles [2]. Several treatments were proposed in the literature. These can be reversible, e.g. braces, physical therapy, stretching, strengthening of the dorsiflexors and botulinum toxin injections (BTX-A) [2]; or non-reversible, e.g. surgical lengthening of the triceps surae and Single Event Multi Level Surgery (SEMLS) [2,3]. Choosing the correct or optimal treatment or each patient is not an easy task, as the success and durability of the treatment ultimately depends on patient's age, conditions, severity of the illness and gait abnormalities [4-6].

Nowadays the walking abnormalities can be assessed by a set of quantitative measurements that provide an objective description of the condition of the patient as well as the effects of the treatment [7-11]. Several studies were conducted on the various treatment options. Computerized gait and its classification by means of synthetic descriptors already demonstrated that SEMLS produces a long term and stable improvement $[3,6],[10]$, the BTX-A has a limited and temporary efficacy but the use of BTX-A is often recommended before surgery $[7,12]$. Due to its non-permanent effect, BTX-A treatment has emerged as a common treatment for the management of spasticity, the drug is well tolerated with minor side effects and the pharmacologic effect is obtained after about 3-6 months [12].

The effect of BTX-A was evaluated by means of quantitative methods in several studies $[7,12,13]$ and the success of such a treatment was correlated to the age of the children, severity of the pathology and type of spasticity [1]. Moreover the response to BTX-A is different depending on subjects and in selected patients it may delay the need for surgery [13].

Recently a new method to classify gait patterns in children with $\mathrm{CP}$, namely the Plantarflexor-Knee Extension Couple Index (PFKE), was developed by Sangeux et al. [14]. This method was based on sagittal ankle and knee scores obtained by gait analysis, and allowed objective identification of the sagittal gait pattern. By using this method, walking patterns of children with $\mathrm{CP}$ can be classified within these groups: (i) True equinus; (ii) Jump gait; (iii) Crouch gait; (iv) Knee recurvatum; (v) Within Normal Limits.

It was observed that subjects may respond differently to a BTX-A treatment depending on their qualitative walking pattern [15]. Moreover, different walking patterns or muscle activation patterns are associated to different pathological conditions of CP. Thus it would be interesting to study the effect and success of a BTX-A treatment on children belonging to different walking pattern groups.

${ }^{*}$ Corresponding author: Andrea Ancillao, Tyndall National Institute, University College Cork, Lee Maltings Complex, Dyke Parade, Cork, Ireland, Tel: +353 212346955; E-mail: andrea.ancillao@hotmail.com

Received July 16, 2018; Accepted August 29, 2018; Published August 31, 2018

Citation: Ancillao A, Galli M, Condoluci C (2018) Efficacy of A-type Botulinum Toxin on Children with Equinus Foot Classified by the Plantarflexor-knee Extension Couple Index: A Retrospective Study. Int J Neurorehabilitation 5: 330. doi: 10.4172/2376-0281.1000330

Copyright: $\odot 2018$ Ancillao A, et al. This is an open-access article distributed unde the terms of the Creative Commons Attribution License, which permits unrestricted use, distribution, and reproduction in any medium, provided the original author and source are credited. 
The aim and strength of the present work, was to evaluate the impact of a specific gait pattern, with respect to the plantarflexor-knee extension couple index, on the effectiveness of the BTX-A treatment. The analysis was conducted through a retrospective study targeting specific joint kinematics and global gait too.

\section{Materials and Methods}

\section{Subjects}

Gait analysis data of children with CP were obtained from the gait database of the Motion Analysis Laboratory of "IRCCS San Raffaele Pisana" Hospital of Rome, Italy. Subjects were selected according to the following criteria: (i) Diagnosis of CP; (ii) spasticity of the triceps surae and equinus foot; (iii) BTX-A treatment; (iv) no previous surgery to the lower limbs; (v) a Gait Analysis evaluation available before and after treatment.

Thirty-three subjects, for a total of 66 lower limbs, were enrolled in the study. Mean age was $9.5 \pm 5.6$ years. GMFCS at the time of diagnosis was within II and III. Gait analysis exams were recorded at the time of diagnosis, i.e. the pre-treatment, and after the treatment. For the cohort of subjects included in this study the post-treatment exam was recorded after and average time of $1.81 \pm 0.87$ months from the treatment. The treatment consisted in BTX-A injections to the triceps surae. In some cases, the subject received also the BTX-A to the tibialis anterior, as decided by the clinician. The goal of the treatment was to improve the overall gait and to improve mobility of the lower limb by controlling spasticity.

Gait analysis data of a control group composed of 18 normally developing children (age: $11.8 \pm 4.7$ years), for a total of 36 lower limbs, served for computing the classification indices.

The study was approved by the Hospital Ethics Committee on 19.04.2017 (n. 14/17) in accordance with the Helsinki Declaration of 1975. All participants were volunteers and their parents or legal guardians gave their written consent to the children's participation.

\section{Equipment}

Gait analysis data were collected by a 12 cameras and 2 force plates optoelectronic system (ELITE 202, BTS Bioengineering, Italy), opportunely calibrated according to manufacturer's instructions. Sampling frequency was $100 \mathrm{~Hz}$. Kinematic data was obtained by solving the Davis protocol [16]. For each subject the results of at least three walking trials were collected and then averaged.

\section{Data processing}

Gait analysis data pre-treatment were classified according to the PFKE [14]. By using sagittal kinematics of ankle and knee, the PFKE indices were computed for the limbs included in the study. The PFKE takes into account the deviation from normality of the ankle and knee angles on the sagittal plane during the mid-stance phase. The mid-stance phase is defined as within the $20 \%$ and $45 \%$ of gait cycle, corresponding to knee extension and ankle dorsiflexion. Knee moment changes from extension to flexion. In this phase, ankle absorbs power through an eccentric contraction of the gastrocnemius complex. PFKE consists of two indices, computed for the ankle and knee:

$$
P_{F K E^{c}}=\frac{1}{45-20+1} \sum_{i=20}^{45} \frac{k_{i}^{c}-\mu_{i}^{c}}{\sigma_{i}^{c}}
$$

where superscript $c$ denotes the knee or ankle curve, subscript $i$ denotes the time instant (in \% of the gait cycle), $\mathrm{k}$ is the value of the kinematic curve at the $i^{\text {th }}$ instant of the gait cycle, $\mu$ is the value of the average kinematic curve of control subjects at the $i^{\text {th }}$ instant and $\sigma$ is the value of the standard deviation from the normal kinematics curve at the $i^{\text {th }}$ instant. e.g. a PFKE index of $(-3,2)$ means that, on average between $20 \%$ and $45 \%$ of the gait cycle, the ankle curve is three standard deviation below normal and the knee curve is two standard deviations above normal [14].

Improvements and variations in gait pattern for each subject were quantitatively evaluated by computing: the Gait Profile Score (GPS) and Gait Variable Scores (GVS) for knee, ankle and hip [17] before and after BTX-A treatment. The spatio-temporal parameters of gait, Stride time, Step length, Step width, Velocity, were also studied before and after treatment in order to check for objective variations in gait pattern after treatment.

Data groups were tested for normality by means of the ShapiroWilk normality test. Since the data were normally distributed, the t-test was applied to study differences in parameters pre $v s$ post treatment. Test was assumed significant if $p<0.05$.

\section{Results}

The subjects were divided in subgroups on the base of PFKE indices. In these subgroups, the analysis was conducted in order to consider how the starting motor phenotype would have effect on the treatment outcomes, taking into account kinematics of the ankle and knee [14]. Three main walking patterns were observed: (i) bilateral true equinus, (ii) bilateral jump gait, (iii) asymmetric gait. The walking and gait parameters of each group were tested for differenced pre $v s$ post BTX-A treatment. The results are briefly depicted trough Figures 1 and 2. For the Gait Variable Scores (GVS) a reduction of the index means an improvement.

\section{Bilateral true equinus}

The subjects with a bilateral true equinus walking pattern were ten, for a total of 20 limbs. The walking pattern of these subjects was further investigated by means of GPS and GVS indices [17] and in terms of spatio-temporal parameters in order to identify changes pre $v s$ post treatment. Detailed indices for bilateral true equinus subjects are shown in Table 1.

\section{Bilateral jump gait}

The subjects with a bilateral jump gait were three, for a total of 6 limbs. The results are shown in Table 2 .

\section{Asymmetric gait}

The subjects with an asymmetric gait were 15, for a total of 30 limbs. The results are shown in Table 3.

\begin{tabular}{|c|c|c|}
\hline & Pre - BTX-A & Post - BTX-A \\
\hline Ankle GVS $\left[^{\circ}\right.$ ] & $15.39(10.82)$ & $10.80(6.47)^{\star}$ \\
\hline Knee GVS $\left[^{\circ}\right]$ & $15.87(5.88)$ & $15.68(6.78)$ \\
\hline Hip GVS $\left[^{\circ}\right]$ & $10.50(6.55)$ & $9.79(4.62)$ \\
\hline GPS $\left[^{\circ}\right]$ & $13.53(4.83)$ & $11.74(2.49)$ \\
\hline Stride Time (ms) & $1163.00(374.33)$ & $1104.50(243.13)$ \\
\hline Step Length $(\mathbf{m m})$ & $357.30(112.68)$ & $388.90(95.87)$ \\
\hline Step Width $\mathbf{( m m})$ & $169.70(58.17)$ & $145.00(55.24)$ \\
\hline Velocity $\mathbf{( m / s )}$ & $0.68(0.30)$ & $0.74(0.23)$ \\
\hline
\end{tabular}

* Significant difference with $p<0.05$

Table 1: Mean and Standard Deviation (SD) of the gait analysis indices computed for the bilateral true equinus. 


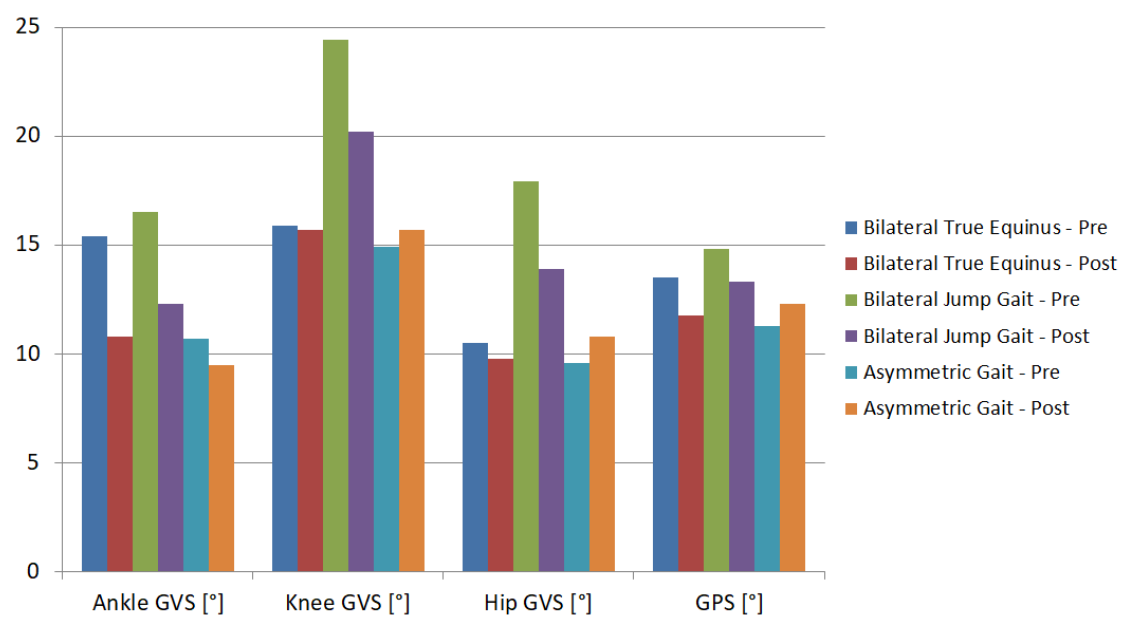

Figure 1: Gait Variable Scores for the three groups studied in this work, pre- and post- treatment.

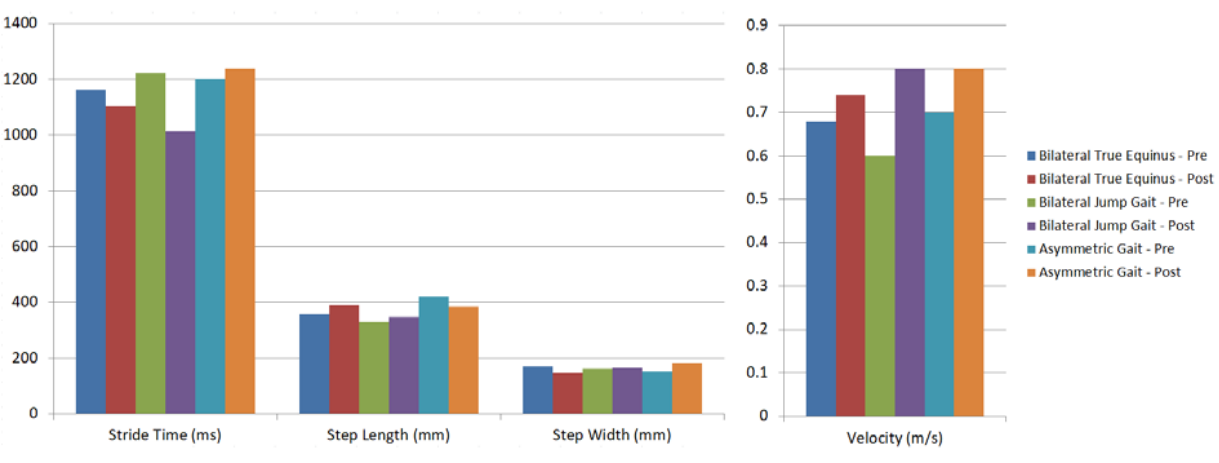

Figure 2: Spatio-temporal gait parameters for the three groups studied and variation pre-post treatment.

\begin{tabular}{|c|c|c|}
\hline & Pre - BTX-A & Post - BTX-A \\
\hline Ankle GVS $\left[^{\circ}\right]$ & $16.5(9.2)$ & $12.3(9.6)^{*}$ \\
\hline Knee GVS $\left[^{\circ}\right]$ & $24.4(7.2)$ & $20.2(7.5)$ \\
\hline Hip GVS [ $\left.{ }^{\circ}\right]$ & $17.9(8.1)$ & $13.9(3.6)$ \\
\hline GPS $\left[^{\circ}\right]$ & $14.8(3.5)$ & $13.3(3.7)$ \\
\hline Stride Time $(\mathbf{m s})$ & $1221.6(423.1)$ & $1013.3(172.1)$ \\
\hline Step Length (mm) & $329.7(102.1)$ & $347.5(95.6)$ \\
\hline Step Width (mm) & $160.9(55.7)$ & $163.8(61.2)$ \\
\hline Velocity (m/s) & $0.6(0.9)$ & $0.8(0.7)$ \\
\hline
\end{tabular}

* Significant difference with $p<0.05$

Table 2: Mean and Standard Deviation (SD) of the gait analysis indices computed for the bilateral jump gait subjects.

\begin{tabular}{|c|c|c|}
\hline & Pre - BTX-A & Post - BTX-A \\
\hline Ankle GVS $\left[^{\circ}{ }^{\circ}\right.$ & $10.7(8.1)$ & $9.5(6.5)$ \\
\hline Knee GVS $\left[^{\circ}\right]$ & $14.9(4.8)$ & $15.7(5.5)$ \\
\hline Hip GVS $\left[^{\circ}\right]$ & $9.6(5.6)$ & $10.8(6.9)$ \\
\hline GPS $\left[^{\circ}\right]$ & $11.3(3.6)$ & $12.3(4.4)$ \\
\hline Stride Time (ms) & $1201.0(215.4)$ & $1239.0(245.8)$ \\
\hline Step Length $(\mathbf{m m})$ & $420.6(96.8)$ & $384.0(118.8)^{*}$ \\
\hline Step Width $(\mathbf{m m})$ & $152.2(52.7)$ & $182.6(67.9)^{*}$ \\
\hline Velocity $(\mathbf{m} / \mathbf{s})$ & $0.7(0.8)$ & $0.8(0.7)$ \\
\hline
\end{tabular}

* Significant difference with $p<0.05$

Table 3: Mean and Standard Deviation (SD) of the gait analysis indices computed for the asymmetric gait subjects.

\section{Discussion}

The "Bilateral True Equinus" and "Bilateral Jump Gait" were the groups that benefitted the most from the treatment at the level of the ankle. In fact, a significant increase in dorsi-plantarflexion capabilities was documented by the Ankle GVS that decreased in the post-treatment (Figure 1 and Table 1). No improvements were observed for the other indices. This effect was observed mainly on the ankle joint and did not produce an improvement in the global quality of gait, as documented by GPS score. No improvements were observed in the spatio-temporal parameters of gait.

These results suggested that children with bilateral true equinus gait pattern received only a minor improvement at the ankle joint, but the overall gait pattern did not benefit from BTX-A treatment.

For the subgroup of asymmetric gait (asymmetric gait means that the two limbs showed different walking patterns) no significant variations of summary measures occurred after treatments and in the GVS of ankle joint was observed, meaning that these subjects have not benefited from the BTX-A treatment. It is important to underline that in this group the initial kinematic condition of ankle joint was less compromised as documented by the ankle GVS.

The results of this work suggested that the effects of BTX-A treatment are not dependent on the PFKE index and the effect was limited to the muscles involved. In our case the main effects were 
observed on the ankle joint and this was in accordance with previous studies [18]. Moreover the effects of the treatment are limited in time and no permanent lengthening of gastrocnemius was observed $[19,20]$. As no effect was observed on the overall gait, BTX-A treatment may be recommended when only a local or temporary effect is desired. The motor phenotype does not influence the overall effect of BTX-A on gait.

The main strength of this study is the analysis of effects of the treatment depending on the motor phenotype classified according to the PFKE index. A limitation of this study was within the relatively low number of subject examined and in the absence of other clinical scales to describe patient's mobility. Furthermore, some subject received the treatment also to the tibialis anterior, in addition to the triceps surae, as the goal of the treatment was to improve lower limb mobility. However, for the aims of the present work, joint/gait functionality was evaluated by means of quantitative gait analysis in order to detect variation between pre- and post-treatment and such measures can be assumed as reliable descriptors of patient's mobility and motor abilities. Further studies should include a larger number of patients, covering also other motor phenotypes.

\section{Conclusion}

The present study focused on the effects of BTX-A on a cohort of subjects classified by means of the PFKE index. Three main walking patterns were observed: (i) bilateral true equinus, (ii) bilateral jump gait, (iii) asymmetric gait. Neither of these groups improved the overall gait after the treatment. The first two groups had an improvement only at the ankle joint. Asymmetric gait walkers did not benefit from the treatment. Thus, based on the results of this study, the BTX-A treatment on toe-walkers subjects is recommended only if a localized and temporary effect is desired. The effects of the treatment on each subject should be monitored by gait analysis and/or other quantitative measures.

\section{Conflict of interests}

The authors declare that there are no known conflicts of interest associated with this publication and there has been no significant financial support for this work that could have influenced its outcome.

\section{Acknowledgments}

The authors would like to commemorate and to give a special thanks to Prof Giorgio Albertini as the incipit of this paper started from him as well as many other papers.

\section{References}

1. Rodda J, Graham HK (2001) Classification of gait patterns in spastic hemiplegia and spastic diplegia: A basis for a management algorithm. Eur J Neurol 8: 98108

2. Ferreira LA, Cimolin V, Costici PF, Albertini G, Oliveira CS, et al. (2014) Effects of gastrocnemius fascia lengthening on gait pattern in children with cerebral palsy using the Gait Profile Score. Res Dev Disabil 35: 1137-1143.

3. Ancillao A, van der Krogt MM, Buizer Al, Witbreuk MM, Cappa P, et al. (2017)
Analysis of gait patterns pre- and post- single event multilevel surgery in children with cerebral palsy by means of offset-wise movement analysis profile and linear fit method. Hum Mov Sci 55: 145-155.

4. Rutz E, Donath S, Tirosh O, Graham HK, Baker R (2013) Explaining the variability improvements in gait quality as a result of single event multi-level surgery in cerebral palsy. Gait Posture 38: 455-460.

5. Rutz E, Baker R, Tirosh O, Romkes J, Haase C, et al. (2011) Tibialis anterior tendon shortening in combination with Achilles tendon lengthening in spastic equinus in cerebral palsy. Gait Posture 33: 152-157.

6. Rutz E, Baker R, Tirosh O, Brunner R (2013) Are results after single-event multilevel surgery in cerebral palsy durable? Clin Orthop Relat Res 471: 10281038.

7. Galli M, Cimolin V, Valente EM, Crivellini M, lalongo T, et al. (2007) Computerized gait analysis of Botulinum Toxin treatment in children with cerebral palsy. Disabil Rehabil 29: 659-664.

8. Ancillao A (2018) Interpretation of gait analysis data by means of synthetic descriptors and a new method for the analysis of the offset. Springer Briefs in Applied Sciences and Technology Pp: 89-121.

9. Vismara L, Cimolin V, Galli M, Grugni G, Ancillao A, et al. (2016) Osteopathic manipulative treatment improves gait pattern and posture in adult patients with prader-willi syndrome. Int J Osteopath Med 19: 35-43.

10. Ancillao A, van der Krogt MM, Buizer Al, Witbreuk MM, Cappa P, et al. (2017) Analysis of gait features variation pre and post SEML surgery in CP by means of GPS and MAP. Gait Posture 49: 120.

11. Vimercati SL, Galli M, Stella G, Caiazzo G, Ancillao A, et al. (2014) Clumsiness in fine motor tasks: Evidence from the quantitative drawing evaluation of children with Down syndrome. J Intellect Disabil Res 59:1-9.

12. Papadonikolakis AS, Vekris MD, Korompilias AV, Kostas JP, Ristanis SE, et al. (2003) Botulinum A toxin for treatment of lower limb spasticity in cerebral palsy Gait analysis in 49 patients. Acta Orthop Scand 74: 749-755.

13. Koman LA, Smith BP, Tingey CT, Mooney JF, Slone S, et al. (1999) The effect of botulinum toxin type $A$ injections on the natural history of equinus foot deformity in paediatric cerebral palsy patients. Eur J Neurol 6: s19-s22.

14. Sangeux M, Rodda J, Graham HK (2015) Sagittal gait patterns in cerebra palsy: The plantarflexor-knee extension couple index. Gait Posture. 41: 586591.

15. Hesse S, Krajnik J, Luecke D, Jahnke MT, Gregoric M, et al. (1996) Ankle muscle activity before and after botulinum toxin therapy for lower limb extensor spasticity in chronic hemiparetic patients. Stroke $27: 455-460$

16. Davis RB, Õunpuu S, Tyburski D, Gage JR (1991)A gait analysis data collection and reduction technique. Hum Mov Sci 10: 575-587.

17. Baker R, McGinley JL, Schwartz MH, Beynon S, Rozumalski A, et al. (2009) The gait profile score and movement analysis profile. Gait Posture 30: 265-269.

18. Galli M, Crivellini M, Santambrogio GC, Fazzi E, Motta F (2001) Short-term effects of 'botulinum toxin A' as treatment for children with cerebral palsy: Kinematic and kinetic aspects at the ankle joint. Funct Neurol 16: 317-323.

19. Scholtes VA, Dallmeijer AJ, Knol DL, Speth LA, Maathuis CG, et al. (2007) Effect of multilevel botulinum toxin a and comprehensive rehabilitation on gait in cerebral palsy. Pediatr Neurol 36: 30-39.

20. Eames NW, Baker R, Hill N, Graham K, Taylor T, et al. (1999) The effect of botulinum toxin $A$ on gastrocnemius length: Magnitude and duration of response. Dev Med Child Neurol 41: 226-232. 